\title{
Les révolutions arabes: révolutions de justice sociale et de liberté
}

Farhad Khosrokhavar

\section{(2) OpenEdition \\ 12 Journals}

\section{Édition électronique}

URL : http://journals.openedition.org/conflits/18213

DOI : $10.4000 /$ conflits. 18213

ISSN : $1777-5345$

Éditeur :

CCLS - Centre d'études sur les conflits lilberté et sécurité, L'Harmattan

\section{Édition imprimée}

Date de publication : 30 décembre 2011

Pagination : 108-112

ISBN : 978-2-296-55700-0

ISSN : 1157-996X

\section{Référence électronique}

Farhad Khosrokhavar, "Les révolutions arabes: révolutions de justice sociale et de liberté », Cultures \& Conflits [En ligne], 83 | Automne 2011, mis en ligne le 04 janvier 2013, consulté le 30 mars 2021. URL: http://journals.openedition.org/conflits/18213 ; DOI : https://doi.org/10.4000/conflits.18213 


\section{Les révolutions arabes: révolutions de justice sociale et de liberté}

\section{Farhad KHOSROKHAVAR}

Farhad Khosrokhavar est professeur à l'École des Hautes Études en Sciences sociales au Cadis. Il a publié plusieurs ouvrages sur l'Iran postrévolutionnaire, l'islam en France et les formes radicales de l'islam en Europe et au Moyen-Orient, notamment en anglais. Ses derniers ouvrages sont : Inside Jihadism: Understanding Jihadi Movements Worldwide, Yale Cultural Sociology Series, Paradigm Publishers, Boulder, 2009 ; Avoir vingt ans au pays des ayatollahs. Vivre dans la ville sainte de Qom (avec Amir Nikpey), Paris, Robert Laffont, 2009. iberté ou justice : peut-on renoncer à la justice pour la liberté ou à la
liberté pour la justice?

Les révolutions arabes, qu'elles aient su renverser le pouvoir (en Tunisie, en Égypte) ou qu'elles soient en crise (au Yémen, en Libye, en Syrie), présentent un double volet : la revendication démocratique et la demande de la justice sociale. Le premier volet a reçu toute la publicité nécessaire, et à juste titre. Mais le second, qui a été la dimension essentielle en Tunisie et, à un moindre titre, important en Égypte, n'a pas reçu toute l'attention qu'il requiert.

Historiquement, il faut bien comprendre que le monde arabe a subi, depuis au moins le début des années 1980, une agression de grande portée : l'introduction du libéralisme à des doses variées, par des politiques dites d'ouverture (infitab). Dans d'autres parties du monde, cette politique a eu certes des aspects négatifs : élargissement du fossé entre riches et pauvres, disparition de pans entiers de l'État Providence, perte des subventions sur les denrées alimentaires de première nécessité pour ceux qui sont économiquement faibles, diminution ou disparition de la sécurité sociale et de l'assurance publique maladie, accentuation des disparités régionales... Mais à côté de cela, des aspects positifs ont pu tout aussi bien être enregistrés : formation d'une bourgeoisie industrialisante, constitution d'une économie plus ou moins compétitive avec embauche d'ouvriers, jonction des pays à économie dite émergente... 
Dans certains pays, avant la période d'ouverture, le rapport société/État était plus ou moins fondé sur un "pacte de sécurité » 1, basé en partie sur l'abandon de revendications politiques en échange d'une relative sécurité de l'emploi et de subventions aux denrées de première nécessité qui permettent un niveau minimal de décence.

Dans une grande partie du monde arabe, rien de tel à échelle comparable. La formation des mafias s'appuyant sur l'État ou sur quelques familles dominantes (en Tunisie les deux familles Ben Ali et Trabelsi, en Égypte la famille du président et ses alliés militaires, au Yemen la famille du président Saleh, en Syrie la famille Assad et celle de leur mère, Makhlouf...) a rendu impossible un «capitalisme sain » et transformé l'ouverture économique en une vaste entreprise d'escroquerie généralisée qui a abouti à l'enrichissement disproportionné de la clientèle de l'État et à l'appauvrissement de vastes couches de la population. Cependant, en Syrie, une «bourgeoisie sunnite » s'est constituée à Damas soutenant plus ou moins Bashar Assad, en Tunisie, une bourgeoisie s'est formée sur les zones côtières promouvant le tourisme sous l'égide de l'État, en Égypte, une dynamique nouvelle s'est instaurée et ces évolutions ont engendré des taux de croissance relativement élevés en Tunisie, en Égypte, voire en Syrie où la croissance a dépassé le $4 \%$ ces dernières années. Néanmoins, l'évolution sociale a été marquée par l'affaiblissement d'importants groupes sociaux qui se sont vus appauvris dans le temps même où ils étaient les témoins indignés de l'enrichissement « indécent 》 des nouveaux parvenus dont beaucoup empochaient, non pas les fruits de leur créativité économique et entrepreneuriale, mais les résultats de leurs liens mafieux avec les détenteurs du pouvoir. Corruption et appauvrissement ont été le lot de franges importantes de la population après ladite «ouverture économique ».

\section{La révolution tunisienne}

La Tunisie est un cas exemplaire : la contestation sociale sous sa forme radicale a commencé en 2008 dans les mines de Gafsa et ne s'est quasiment pas interrompue, malgré l'allégeance du leadership national de l'UGTT au pouvoir en place. La révolte de Ben Guerdane en 2010, zone frontalière avec la Libye où les commerçants locaux vivant dans une région sous-développée ont vu l'imposition par le régime libyen de taxes sur leurs biens d'exportation, témoigne du même constat d'indignation et de révolte contre l'appauvrissement. L'étincelle qui a mis le feu aux poudres a été l'auto-immolation de Bouazizi dans la ville de Sidi Bouzid située dans cette partie laissée pour compte de la Tunisie de l'intérieur qui contrastait avec les régions côtières où s'étalait l'aisance matérielle pour les touristes et où existaient des possibilités d'emploi pour la population locale. Ce n'est pas tant l'absence de démocratie au sens formel du terme, mais l'injustice sociale, criarde, tangible au quotidien,

1. Hibou B., La Force de l'Obéissance, Paris, La Découverte, 2006. 
qui a été la cause de la contestation sociale à ses débuts. Le mouvement qui s'est déclenché et qui a ensuite atteint la Tunisie entière s'est fondé sur les réseaux des "diplômés chômeurs ", sur les membres du syndicat des enseignants primaires, ceux de la santé et des télécommunications, sur les membres intermédiaires de l'UGTT, tous regroupés au sein dudit «comité des marginaux ». Il y a une rupture de fait entre la direction nationale de l'UGTT et les sections régionales, acquises à la contestation et à sa politisation. Dans la ville de Kasserine qui joue un rôle clé dans la révolution tunisienne, les avocats sont les leaders du mouvement. En effet, le 24 décembre, les avocats viennent rejoindre le mouvement et manifestent dans la rue, le Conseil national des avocats devenant pour un moment le leader du mouvement national. S’organisent alors des manifestations dans différentes villes dont Tunis, Sousse, Monastir, Fafsa... ${ }^{2}$

Dans toutes ces manifestations, la jeunesse «netizenisée » arrive en masse et, par les réseaux de Facebook et de Twitter, se constituent alors des rassemblements de plus en plus étendus. La Tunisie a un taux de pénétration de l'internet qui est relativement élevé (34\% de la population) ${ }^{3}$. La revendication démocratique s'articule sur la demande de justice sociale et en un sens, elle en est la conséquence : dans un système mafieux et hermétiquement fermé comme celui de Ben Ali, aucune justice sociale ne pouvait se réaliser sans remettre en cause le système politique, pas simplement autoritaire, mais total, voire totalitaire sous certains aspects. La demande de justice sociale, après l'échec des expériences précédentes, débouche sur une revendication politique qui devient « naturellement » démocratique. Il est vrai que les expériences précédentes avec le nationalisme autoritaire et le cas algérien avec l'islamisme radical ont servi à détrôner toute vision utopique « holistique » du politique et ont abouti à une version plus ouverte. Le parti se réclamant des Frères musulmans $(E n-N a b d a)$ a su aussi prendre en considération la dimension démocratique comme essentielle et a mis en sourdine la revendication de « l'État Islamique ».

Depuis le renversement du régime Ben Ali, les revendications de justice sociale se trouvent plus ou moins en crise, faute de modèle établi. Autant la « démocratie » formelle dispose d'un modèle relativement charpenté, autant la demande d'une "société juste » se trouve en état de déshérence, compte tenu de la prévalence de l'État libéral en Occident où les démocrates américains et la gauche européenne se trouvent sur la défensive devant la dynamique nouvelle lancée par les « pays émergents » et leur main d'œuvre bon marché. N'empêche, les revendications ouvrières se trouvent plus ou moins encouragées, ne serait-ce que par la possibilité d'ériger des syndicats et des partis politiques nouveaux.

2. Crisis Group, «Soulèvements populaires en Afrique du Nord et au Moyen-Orient : (IV) La voie tunisienne ", Rapport Moyen-Orient/Afrique du Nord, n¹06, 28 avril 2011.

3. Voir Internet World Stats http://internetworldstats.com/ Page consultée le 3.10.2011 


\section{La révolution égyptienne}

Le cas égyptien est tout aussi instructif. Le Mouvement du 6 avril qui débuta en 2008, se donnait pour but de venir en aide aux salariés de la cité ouvrière de Mahalla al Kubra, ville située en plein milieu du delta du Nil avec une industrie textile et une population d'un demi-million d'habitants. Le 6 avril 2008, en réaction contre l'augmentation du coût de la vie, notamment des produits alimentaires, un appel à la grève entraîne l'arrestation de 33 personnes. Le Mouvement 6 avril était lui-même animé par des non-ouvriers, notamment Asma Mahfouz, une jeune femme qui jouera par la suite un rôle déterminant dans la révolution de 2011, Ahmad Maher, une autre figure de proue de la place Tahrir, ainsi qu'Issa Abdel Fatah. Ce mouvement se greffa donc sur un mouvement ouvrier mais s'en détacha en s'autonomisant par le biais de Facebook qui permit l'adhésion de quelque 70000 jeunes à la contestation.

Durant les trois jours précédant la démission de Mubarak, le 11 février 2011, les ouvriers égyptiens apportèrent leur contribution au mouvement révolutionnaire en lançant une soixantaine de grèves dont certaines assorties de revendications explicitement politiques ${ }^{4}$. Par la suite, le mouvement revendicatif s'est poursuivi et près de 200 mouvements d'action collective ont été tentés au cours des six premiers mois de 2011, ce qui correspondait au nombre total des mouvements depuis $2004^{5}$ et ce qui a permis aux travailleurs d'avoir gain de cause sur le front des salaires.

L'acquis le plus important de la révolution du 11 février est la constitution de syndicats et de partis politiques indépendants. Le syndicat national égyptien, le ETUF (Egyptian Trade Union Federation) était, depuis sa constitution en 1957 sous la domination de l'État autocratique mais, en 2009, sous la direction de Kamal Abu Aita, se constitua une branche nouvelle qui s'est voulue indépendante de l'ETUF : la Reta (Real Estate Tax Authority Union). Les prémices remontent à 2006 lorsque les ouvriers commencèrent à faire grève dans l'ensemble du pays ; puis en 2007 Abu Aita organisa une grève à laquelle participèrent une cinquantaine de milliers d'ouvriers ; il monta alors 29 comités de grève, un dans chaque district régional et un comité central au Caire ; les grévistes purent ainsi obtenir des augmentations substantielles de salaire et on décida de fonder la Reta. Mais ETUF déposa une plainte contre la nouvelle organisation, obtenant des dommages et intérêts contre elle. Alors même que les membres de l'ETUF réprimaient les manifestations de la Place Tahrir lors de la révolution, ceux de la Reta y participaient à côté des autres groupes révolutionnaires ${ }^{6}$. Depuis la Révolution du 11 février, huit syndicats ouvriers se

4. Beinin J., "What have workers gained from Egypt's revolution?”, Foreign Policy, 20 juillet 2011.

5. Ibid.

6. International Trade Union Confederation, Interviews, Gros Plan sur Kamal Abou Aita (RETA-Egypte), 7 mars 2011. 
sont constitués à Sadat City où 50000 travailleurs sont employés par 200 entreprises dans le textile, l'acier et le fer. Des syndicats, comme celui du transport urbain, sont également puissants et leur organisation fait de plus en plus entendre sa voix. Depuis la révolution, les syndicats ouvriers ont pu imposer une augmentation du salaire minimum.

Alors que dans le modèle tunisien les syndicats et les groupes de travailleurs ont joué un rôle de premier plan, dans la révolution égyptienne ce rôle a été plus indirect, se cantonnant aux ouvriers en tant qu'individus plutôt qu'aux organisations, encore sous la tutelle de l'État autoritaire. Mais c'est juste avant la révolution et après, que leur rôle s'est accru.

En tout état de cause, avant la période révolutionnaire proprement dite, beaucoup de mouvements, même celui du 6 avril, ont lourdement insisté sur l'importance capitale de la justice sociale.

\section{La justice sociale comme constitutive du Printemps arabe}

La dimension anti-libérale (au sens du libéralisme économique) des révolutions majeures du Printemps arabe, à savoir les révolutions tunisienne et égyptienne, est, pour ainsi dire, manifeste, à des degrés divers il est vrai. Dès lors, pour une grande partie des acteurs de ces révolutions, la démocratie se conjugue de pair avec la justice sociale, l'idéal de la liberté politique étant indissociable, dans cette perspective, de celui d'une société mettant fin à des disparités économiques trop élevées. Ce n'est certainement pas la première fois que les exigences de liberté et d'égalité sont posées ensemble, voire postulées comme indissociables chez une partie importante des acteurs révolutionnaires. La Révolution française tentait de concilier les deux, non sans difficulté, et surtout, non sans violence. Les révolutions majeures de la première moitié du XXe siècle (révolutions russe ou chinoise) ont sacrifié la liberté à un certain égalitarisme dans la pénurie. Par la suite, la Révolution iranienne de 1979, à ses débuts, se voulait celle des "déshérités ", mais ensuite, le déni de liberté et de justice sociale s'est conjugué dans un système théocratique répressif. Les révolutions de velours (ou de couleur) en Europe de l'Est ont prôné la démocratie, mais la justice sociale était, au mieux, un idéal subordonné. Les révolutions arabes se posent la même question avec acuité. Il s'agit peut-être de la quadrature du cercle, mais encore une fois, il demeure impossible de séparer une exigence de l'autre, sous peine de manquer à l'idéal démocratique lui-même. Cependant, faute de modèle crédible et vu l'ascendant du modèle néo-libéral, l’idéal de la justice sociale risque d'être le premier à être sacrifié dans la crise post-révolutionnaire. 